YCTP-P41-92

\title{
On the Limits of Chiral Perturbation Theory
}

\author{
Thomas Appelquist and John Terning \\ Department of Physics, Yale University, New Haven, CT 06511
}

October 2, 2018

\begin{abstract}
We consider the relation between the breakdown scale of chiral perturbation theory, $\Lambda_{\chi}$, for large values of $N$ (flavor), and the scale associated with "new" physical thresholds. This question is addressed using both the linear $\sigma$ model and an asymptotically-free gauge theory to describe the high energy dynamics. It is suggested that the massive physical threshold could be well above $\Lambda_{\chi}$.
\end{abstract}

\section{Introduction}

In a recent paper [1], Soldate and Sundrum made an interesting observation about chiral perturbation theory; especially as applied to technicolor theories with a large number, $N$, of technifermions. They showed that in the large $N$ limit, chiral perturbation theory (a low-energy expansion) breaks down at a scale no higher than $\Lambda_{\chi} \approx 4 \pi f / \sqrt{N}$, where $f$ is the Nambu-Goldstone boson (NGB) decay constant. Chivukula, Dugan, and Golden [2] have pointed out that this could imply the existence of physical states with masses of order $\Lambda_{\chi}$ or lower, a scale much smaller than $4 \pi f$ for large $N$. If chiral perturbation theory applies all the way up to $\Lambda_{\chi}$, then strong interactions will have set in, and it is therefore difficult to study this problem in detail without a strong-coupling computation. In this paper we will 
instead examine a specific, and very simple, model in order to further elucidate the question of the existence of low-lying thresholds. We will then give a more general argument indicating that there may not need to be physical states with masses near $\Lambda_{\chi}$. We will conclude with some remarks on the implications for technicolor models.

\section{Estimating the Coefficients in a Chiral Lagrangian}

We first discuss methods for estimating the size of coefficients in an $S U(N) \otimes$ $S U(N)$ chiral Lagrangian. Recall that, in the derivative expansion, the leading two-derivative term is:

$$
\mathcal{L}_{2}=\frac{f^{2}}{4} \operatorname{Tr}_{\mu} U^{\dagger} \partial^{\mu} U
$$

where

$$
U=\exp \left(\frac{2 i \pi^{a} T^{a}}{f}\right)
$$

and $T^{a}$ is a generator of $S U(N)$ normalized so that $\operatorname{Tr}\left(T^{a} T^{b}\right)=\frac{1}{2} \delta^{a b}$. Note that $\mathcal{L}_{2}$ is characterized by a single parameter $f$, giving a dimensionless $\pi \pi$ scattering amplitude proportional to $p^{2} / f^{2}$, where $p$ represents the external momenta. At $O\left(p^{4}\right)$, there are various terms in $\mathcal{L}_{4}$ with a-priori unknown coefficients. One often uses the estimate that the contributions from $\mathcal{L}_{4}$ should be the same order of magnitude as the radiative corrections from NGB loops [3, 4, 5]. Up to factors of $N$, these

corrections will typically be of order $p^{2} /(4 \pi f)^{2}$ relative to the $\mathcal{L}_{2}$ contribution. (At the one-loop level, this can be corrected by a logarithm of an ultraviolet cutoff $\Lambda$ over an infrared scale $\mu$.)

To see how this estimate arises, suppose that the natural scale of the underlying physics driving the symmetry breakdown is denoted by $\Lambda_{s b}$. The "heavy" 
physics above this scale is integrated out, generating an effective low-energy theory, the chiral Lagrangian, for the degrees of freedom, the NGB's, relevant below $\Lambda_{s b}$. (We are primarily interested here in the case of "dynamical" symmetry breaking, in which the underlying physics is strongly interacting. The possibility that the underlying physics is weakly coupled, as in a weakly coupled linear $\sigma$ model, will also be considered briefly in section 3.) All the coupling constants in this chiral Lagrangian are renormalized at the matching scale $\Lambda_{s b}$. Suppose, however, that we want to renormalize at a somewhat lower scale $\mu$, by integrating out the Nambu-Goldstone degrees of freedom between $\Lambda_{s b}$ and $\mu$. Then the coefficients in $\mathcal{L}_{4}$ are renormalized by these NGB loops. Assuming that the contribution from physics above $\Lambda_{s b}$ does not dominate or cancel the NGB contribution, this leads to the above estimate $\left(\mathrm{O}\left(p^{2} /(4 \pi f)^{2}\right)\right)$ for the order of magnitude (relative to $\left.\mathcal{L}_{2}\right)$ of the various terms in $\mathcal{L}_{4}$. This simple notion is known as "naturality" [3], or "naive dimensional analysis" 田, 战.

There are two issues that can complicate this simple picture. First, the two contributions mentioned above can have a different dependence on the underlying parameters of the theory. For example, in QCD, they can depend differently on the number of colors, $N_{c}$. The NGB loop computation is independent of $N_{c}$ (if $f$ is held fixed), whereas large $N_{c}$ arguments [6, 7] suggest that the leading contribution from above $\Lambda_{s b}$ is proportional to $N_{c}$. This is not surprising since the two, additive, contributions come from different physics in different energy regimes. A more subtle problem is that it may not be possible to calculate the entire pion loop contribution within the framework of chiral perturbation theory. This is the case when chiral perturbation theory fails at a scale $\Lambda_{\chi} \ll \Lambda_{s b}$. We know that chiral perturbation 
theory must fail when energies of order $\Lambda_{s b}$ are reached since there is "new" physics there that has been integrated out. However, there is no guarantee that chiral perturbation theory converges for the entire range of energies below this scale. In this paper we will study the relation between the two scales $\Lambda_{s b}$ and $\Lambda_{\chi}$.

\section{$3 \quad$ A Linear $\sigma$ Model}

In order to gain a better understanding of what is happening with the chiral Lagrangian, it is helpful first to consider a renormalizable model that reduces to the chiral Lagrangian when some of the particles become heavy. For $N=2$, the simplest such model is the $O(4)$ linear $\sigma$ model. This model, when it undergoes spontaneous symmetry breaking (the symmetry of the vacuum being $O(3)$ ), reduces to an $O(4)$ nonlinear $\sigma$ model (which is equivalent to the $S U(2) \otimes S U(2)$ chiral Lagrangian) in the low-energy (or $M_{\sigma} \rightarrow \infty$ ) limit. The relation between the linear and nonlinear theories has been explored in some detail (see ref. [8] and further references therein).

The linear $\sigma$ model that reduces to the $S U(N) \otimes S U(N)$ chiral Lagrangian has been studied by several authors [9, 10]. We briefly summarize the properties of such a model. Consider the $U(N)_{L} \otimes U(N)_{R}$ linear $\sigma$ model:

$$
\mathcal{L}=\frac{1}{2} \partial_{\mu} M^{\dagger} \partial^{\mu} M-\frac{\lambda}{4}\left[\operatorname{Tr} M^{\dagger} M M^{\dagger} M+\frac{\alpha}{N}\left(\operatorname{Tr} M^{\dagger} M\right)^{2}-2 f^{2} \operatorname{Tr} M^{\dagger} M\right],
$$

where

$$
M=\frac{\sigma+i \eta^{0}}{\sqrt{N}} I+\sqrt{2}\left(\Sigma^{a}+i \pi^{a}\right) T^{a}
$$

$M$ transforms as

$$
M \rightarrow L M R^{\dagger}
$$


where $L$ and $R$ are elements of $U(N)_{L}$ and $U(N)_{R}$ respectively. The $\Sigma$ 's and $\pi$ 's are scalars and pseudoscalars respectively, and transform as adjoints under $S U(N)_{V}$. The $\sigma$ and $\eta^{0}$ are a scalar and a pseudoscalar respectively, and are singlets under $S U(N)_{V}$. The $\eta^{0}$ and $\pi$ 's are massless NGB's. Note that the $\eta^{0}$ is an "axion", corresponding to the spontaneously broken $U(1)_{A}$ symmetry. A term proportional to $\operatorname{det} M+\operatorname{det} M^{\dagger}$ could be introduced in the Lagrangian, which would give a mass to the "axion", but then the model would not be renormalizable for $N>4$. However, since we are interested mainly in scattering amplitudes for the $\pi$ 's, the "axion" will only contribute through loop effects, and "axion" loops are suppressed relative to $\pi$ loops by at least one factor of $1 / N$. Thus they can be safely ignored for large $N$.

At tree level, the condition for minimizing the vacuum energy is:

$$
0=\left.\frac{\partial V}{\partial M_{i j}}\right|_{M=M_{0}}=\frac{\lambda}{2}\left[M_{0}^{\dagger} M_{0} M_{0}^{\dagger}+\left(\frac{\alpha}{N} \operatorname{Tr} M_{0}^{\dagger} M_{0}-f^{2}\right) M_{0}^{\dagger}\right]_{j i},
$$

where $M_{0}$ is the vacuum expectation value of $M$ at tree level. The trivial solution $M_{0}=0$ is unstable for $f^{2}>0$. The other solution is:

$$
M_{0} M_{0}^{\dagger}=M_{0}^{\dagger} M_{0}=f^{2}-\frac{\alpha}{N} \operatorname{Tr} M_{0}^{\dagger} M_{0}
$$

Thus we see that $M_{0}$ is proportional to a unitary matrix, and hence, by a symmetry transformation, $M_{0}$ can be made proportional to the identity, i.e. $M_{0}=v I$. With this choice of basis, we have from Eqs. (4) and (7),

$$
\sigma_{0}=\sqrt{N} v=\sqrt{\frac{N}{1+\alpha}} f
$$

So, at tree order, the $U(N)_{L} \otimes U(N)_{R}$ symmetry is spontaneously broken down to $U(N)_{V}$. To do perturbation theory in the linear model, we shift the field,

$$
M \rightarrow M+v
$$


Expanding out the terms in the Lagrangian, the masses of the $\sigma$ and the $\Sigma$ 's at tree level are given by: $M_{\sigma}^{2}=2 \lambda f^{2}$, and $M_{\Sigma}^{2}=2 \lambda v^{2}$. As promised, the $\eta^{0}$ and $\pi$ 's are massless NGB's. For energies much below $M_{\sigma}$, this model reduces to the nonlinear $U(N) \otimes U(N) \sigma$ model, that is, a chiral Lagrangian supplemented to include the effects of an "axion".

Consider this chiral Lagrangian in the large $N$ limit, with $\lambda$ and $f$ held fixed. As shown in refs. [1, 2], the partial wave $\pi \pi$ scattering amplitude in the singlet channel violates unitarity at energies of order $\Lambda_{\chi}=4 \pi f / \sqrt{N}$, and chiral loop corrections become of order one at this same scale. Thus, chiral perturbation theory breaks down at the scale $\Lambda_{\chi}$ (or lower), but how is this scale related to the lowest non-NGB threshold in this model? One would expect this threshold to be at the $\sigma$ and $\Sigma$ thresholds, at $\sqrt{2 \lambda} f$ and $\sqrt{2 \lambda} v$. For what might be thought to be moderately strong coupling $\left(\lambda \approx 4 \pi^{2}\right)$, this is much larger than $\Lambda_{\chi}$. To understand this difference, it is helpful to return to the linear model.

We begin with some comments on the loop expansion in the underlying linear model. First note that diagrams with the highest powers of $N$ come from graphs with $\pi$ and $\Sigma$ loops. The large $N$ analysis for these diagrams is similar to that for QCD, i.e., since the $\pi$ 's and $\Sigma$ 's are in adjoint representations the counting can be simplified by using the double-line notation [11]. One can show that at $L$ loops the leading graphs are of order $N^{L}$. Thus the loop expansion parameter is roughly $\lambda N / 4 \pi^{2}$, and the expansion can be expected to break down, defining the onset of strong coupling, when this parameter becomes of order unity. 円

If one demands that the loop expansion parameter in the linear model be of

\footnotetext{
${ }^{1}$ Here $\lambda$ is defined at scales of order $M_{\sigma}$.
} 
order unity or smaller, then $M_{\sigma}=\sqrt{2 \lambda} f \leq 2 \sqrt{2} \pi f / \sqrt{N} \approx \Lambda_{\chi}$. In this case, the massive physical threshold is of order $\Lambda_{\chi}$ or smaller. At the onset of strong coupling $\left(\lambda N / 4 \pi^{2}=O(1)\right)$, the threshold is of order $\Lambda_{\chi}$. It is only in the weak coupling case, $\lambda N / 4 \pi^{2} \ll 1$, which is not of central interest here, that the physical threshold will fall well below $\Lambda_{\chi}$.

Suppose, next, that $\lambda \gg 4 \pi^{2} / N$, so that the loop expansion is useless, and the tree level relation $M_{\sigma}=\sqrt{2 \lambda} f$ is unreliable. Here, we imagine defining $\lambda$ at a scale on the order of the threshold for the production of the massive states of the theory, whatever they may be. Chiral perturbation theory will breakdown at $\Lambda_{\chi}$, as it must, involving the same factors of $N$ that cause the breakdown of the loop expansion in the linear model. Since the underlying problem is simply the large number of diagrams, however, this gives us no direct information about finitemass singularities of the scattering amplitudes in momentum space, and hence no direct information about the masses of the $\sigma$, the $\Sigma$ 's, or any other possible massive physical states. The mass scale of new thresholds cannot be computed in either chiral perturbation theory or the underlying linear model.

To summarize the discussion of the linear $\sigma$ model as a description of the high energy dynamics, the massive physical threshold will be below $\Lambda_{\chi}$ when the loop expansion parameter is small, and it will be roughly at $\Lambda_{\chi}$ when this parameter is $O(1)$. The threshold could, however, be above $\Lambda_{\chi}$ if the loop expansion parameter ( defined at scales on the order of the threshold) is larger than unity. In either of the latter two cases, the breakdown of chiral perturbation theory at $\Lambda_{\chi}$ is a reflection of the breakdown of the loop expansion. In the next section we will comment more generally on the possibility that the massive threshold could be well above $\Lambda_{\chi}$ by 
briefly returning to a more physical discussion of the low-energy chiral Lagrangian.

\section{Beyond the Linear $\sigma$ Model and Beyond the Loop Expansion}

As pointed out in ref. [2], the most general $\pi^{a} \pi^{b} \rightarrow \pi^{c} \pi^{d}$ scattering amplitude for the $S U(N) \otimes S U(N)$ chiral Lagrangian can be written as:

$$
\begin{aligned}
a(s, t, u)^{a, b ; c, d}= & \delta^{a b} \delta^{c d} A(s, t, u)+d^{a b e} d^{c d e} B(s, t, u) \\
& +(s \leftrightarrow t, b \leftrightarrow c)+(s \leftrightarrow u, b \leftrightarrow d),
\end{aligned}
$$

where $d^{a b c}$ is defined by $\left\{T^{a}, T^{b}\right\}=d^{a b c} T^{c}+\delta^{a b} / N$.

Now if we choose any functions for $A(s, t, u)$ and $B(s, t, u)$ that are consistent with chiral symmetry and unitarity, there should be a chiral Lagrangian (with an infinite number of terms) that reproduces our arbitrary scattering amplitude when an exact calculation is performed 谏. The constraint that chiral symmetry imposes on the two functions is that the leading low energy behavior (as $s, t$, and $u \rightarrow 0$ ) agrees with the tree order result [2] derived from Eq. (11):

$$
\begin{aligned}
A(s, t, u) & =\frac{2 s}{N f^{2}}+\ldots \\
B(s, t, u) & =\frac{s}{f^{2}}+\ldots
\end{aligned}
$$

Suppose that $A$ and $B$ are functions whose only singularities are cuts corresponding to multi- $\pi$ thresholds, i.e., there are no resonances. There is some chiral Lagrangian, consisting of Eq. (1) and higher-order terms, that can produce such a scattering amplitude. Of course, chiral perturbation theory, at any finite order, will be useless for energies above $\Lambda_{\chi}=4 \pi f / \sqrt{N}$, since the expansion parameter $p^{2} / \Lambda_{\chi}^{2}$ 
is larger than one. However, when the chiral perturbation series is summed to all orders, the functions $A$ and $B$ can be obtained, even for $p^{2} \gg \Lambda_{\chi}^{2}$. This could still be well below the scale of "new" physics. This is reminiscent of the fact that some series can converge, even when the expansion parameter is larger than one, e.g., the Taylor expansion of $\left(p^{2} / f^{2}\right) e^{-p^{2} / \Lambda_{\chi}^{2} \ln \left(-a p^{2} / \Lambda_{\chi}^{2}\right)}$ (where $a$ is some constant) when the exponential is expanded in powers of $p^{2} / \Lambda_{\chi}^{2} \ln \left(-a p^{2} / \Lambda_{\chi}^{2}\right)$.

This type of behavior, with no massive physical threshold at all, is, of course, extreme. It would require much cancellation in the chiral loop expansion in order to obtain the requisite inverse factorials. However, between the two extremes of thresholds at $\Lambda_{\chi}$, and no thresholds, there lies a range of possibilities for the threshold mass. Reality may lie somewhere in this range. To say this in another way, the actual form of the scattering amplitude is almost surely not as simple as the above exponential form. In particular, terms of order $\left(p^{2}\right)^{n}$ in the low energy expansion will naturally come multiplied by various powers of $\ln \left(-p^{2} / \Lambda_{\chi}^{2}\right)$, i.e. $\ln ^{m}\left(-p^{2} / \Lambda_{\chi}^{2}\right)$, where $m=0,1, \ldots n-1$. Even so, the fact that $\Lambda_{\chi}$ may set the scale for all the terms in chiral perturbation theory does not necessarily mean that there must be a massive physical threshold at $\Lambda_{\chi}$.

\section{$5 \quad$ Implications for Technicolor}

The motivation for studying theories with large numbers of flavors comes from technicolor model building. There, for example, a popular class of models has one family of technifermions, and hence an $S U(8) \otimes S U(8)$ flavor symmetry. In technicolor the question of the how $\Lambda_{\chi}=4 \pi f / \sqrt{N}$ is related to the scale of "new" physics can be rephrased as: what is the relation between $f$ and the technicolor 
confinement scale? (The confinement scale $\Lambda_{t c}$, is roughly the scale of "new" physics in technicolor theories, and $f$ is related to the weak scale by $f=250 \mathrm{GeV} \sqrt{2 / N}$.) A typical procedure for estimating $f$ relies on the approximation that $f$ is determined by diagrams with only one fermion loop. This is reliable in large- $N_{c}$ QCD, and is also assumed in Pagels-Stokar type analyses. These analyses lead to the rough estimate $4 \pi f \approx \sqrt{N_{t c}} \Lambda_{t c}$, where $N_{t c}$ is the number of technicolors. This would place $\Lambda_{\chi}$ well below $\Lambda_{t c}$ for $N$ well above $N_{t c}$.

With $N$ large, however, these one-fermion-loop analyses are not likely to be reliable. Additional technifermion loops enter with a factor of $N$, and could be at least as important as the gauge corrections to the single fermion loop. Even with $N$ constrained to preserve the asymptotic freedom of the theory (as in the above one-family model with $N_{t c}$ even as low as 2), it could be that contributions with arbitrary numbers of fermion loops are equally important. Among all these contributions, there will appear, of course, the low energy loops of NGB's, that can be dealt with (chiral)-perturbatively below $\Lambda_{\chi}$, but give no direct information about the relation of $4 \pi f$ to the confinement scale $\Lambda_{t c}$. The contributions from momenta above $\Lambda_{t c}$, on the other hand, do provide this connection, but may involve an $N$ dependence that renders the above Pagels-Stokar estimate invalid. Whether the correct result lowers the scale of "new" physics toward $\Lambda_{\chi}$ or leads to an even larger gap between the two scales is not clear to us.

In order to get a more physical picture of what may be going on in such theories, imagine integrating out the "heavy" physics in an asymptotically-free technicolor theory. Since a low-energy expansion must be performed in order to obtain the chiral Lagrangian, the mass that sets the scale for $f$ (and hence $\Lambda_{\chi}$ ) in the 
low-energy theory is the mass (actually the zero-momentum mass) of the "lightest" non-NGB, e.g. the techni- $\rho$. For particles with widths of the same order as their masses (as would be expected, for example, for a techni- $\rho$ in a theory with $N$ as large as 8), there is no simple connection between the zero-momentum mass and the physical mass. Thus, it is reasonable to expect that there may not be a direct connection between $\Lambda_{\chi}$ and the location of the lowest resonance peak, i.e. the scale of "new" physics.

The estimates for $S$ and $T$ in technicolor models suffer from the same uncertainties as the estimates of $f$, since all current methods for estimating $S$ and $T$ [7, 12] rely either on scaling QCD (where the quark loop expansion presumably does work) up to technicolor (where the technifermion loop expansion may or may not work), or on an explicit single technifermion loop computation. In particular, estimates of $S$ based on the techni- $\rho$ mass may be unreliable since there is no simple connection between $f$ and the physical techni- $\rho$ mass in large $N$ technicolor models.

\section{Conclusions}

We have suggested that the fact that chiral perturbation theory for the $S U(N)$ $\otimes S U(N)$ chiral Lagrangian breaks down at very low energies $(\approx 4 \pi f / \sqrt{N})$ for large $N$ does not necessarily indicate unexpected, low-lying, physical states. We have noted that the potential breakdown of the technifermion loop expansion (for large $N$ ) for the estimation of $f, S$, and $T$ in terms of the parameters of a technicolor theory, reflects the difficulty of relating the chiral symmetry breakdown scale to the threshold for "new" physics. 


\section{Acknowledgments}

We thank S. Chivukula and R. Sundrum for helpful discussions. JT acknowledges the financial support of a Superconducting Super Collider fellowship from the Texas National Research Laboratory Commission. This work was supported in part by the U.S. Department of Energy under contracts DE-AC-02-76ERO3075 and DE-FG-02-84ER40153.

\section{References}

[1] M. Soldate and R. Sundrum, Nucl. Phys. B340 (1990) 1.

[2] R. Chivukula, M. Dugan, and M. Golden, Harvard preprint HUTP-92/A025, hep-ph/9206222.

[3] T. Appelquist in Gauge Theories and Experiments at High Energies, Ed. by K.C. Brower and D.G. Sutherland, Published by the Scottish University Summer School in Physics, St. Andrews (1980);

[4] S. Weinberg, Physica 96A (1979) 327.

[5] H. Georgi and A. Manohar, Nucl. Phys. B234 (1984) 189; H. Georgi and L. Randall, Nucl. Phys. B276 (1986) 241.

[6] J. Gasser and H. Leutwyler, Nucl. Phys. B250 (1985) 465.

[7] M. Peskin and T. Takeuchi Phys. Rev. D46 (1992) 381. 
[8] D. Bessis and J. Zinn-Justin, Phys. Rev. 5 (1972) 1313; T. Appelquist and C. Bernard, Phys. Rev. D22 (1980) 200; Phys. Rev. D23 (1981) 425; J. van der Bij and M. Veltman, Nucl. Phys. B231 (1984) 205.

[9] W. Bardeen and B. Lee, Phys. Rev. 177 (1969) 2389; M. Lévy, Nuovo Cim. 52A (1967) 23.

[10] A. Paterson, Nucl. Phys. B190 (1981) 188; H. Geddes, Phys. Rev. D20 (1979) 531.

[11] G. t'Hooft Nucl. Phys. B72, (1980) 461; S. Coleman, Aspects of Symmetry, Cambridge University Press, Cambridge (1985).

[12] T. Appelquist, T. Takeuchi, M. Einhorn, and L.C.R. Wijewardhana, Phys. Lett. B 232, (1989) 211; M. Golden and L. Randall, Nucl. Phys. B361, (1991) 3; B. Holdom and J. Terning, Phys. Lett B 247, (1990) 88; M. Peskin and T. Takeuchi, Phys. Rev. Lett. 65, (1990) 964; R. Johnson, B.-L. Young, and D. McKay, Phys. Rev D43, (1991) R17; A. Dobado, D. Espriu, and M. Herrero, Phys. Lett. B253, (1991) 161; R. Sundrum and S. Hsu, Lawrence Berkeley Lab. preprint LBL-31066 hep-ph/9206225; T. Appelquist and G. Triantaphyllou, Phys. Lett. B 278, (1992) 345; R. Chivukula, M. Dugan, and M. Golden, Boston University preprint BUHEP-92-25, hep-ph/9207249. 